\title{
The Location of Multifidus Atrophy in Patients With a Single Level, Unilateral Lumbar Radiculopathy
}

\author{
Jung-Il Kang, MD ${ }^{1}$, Sun-Yu Kim, MD², Jin-Hyun Kim, MD ${ }^{1}$, Hyun Bang, MD ${ }^{1}$, In-Sik Lee, MD, PhD ${ }^{1}$
}

\begin{abstract}
${ }^{1}$ Department of Rehabilitation Medicine, Konkuk University Medical Center, Konkuk University School of Medicine, Seoul;
\end{abstract}
${ }^{2}$ Champyunhan Pain and Rehabilitation Clinic, Cheongwon, Korea

Objective To identify the correlations between the location of multifidus atrophy and the level of lumbar radiculopathy.

Methods Thirty-seven patients who had unilateral L4 or L5 radiculopathy were divided into 2 groups; the L4 radiculopathy (L4 RAD) group and the L5 radiculopathy (L5 RAD) group. Bilateral lumbar multifidus muscles at the mid-spinous process level of L4 vertebra (L4 MSP), the mid-spinous process level of L5 vertebra (L5 MSP), and the mid-sacral crest level of S1 vertebra (S1 MSC) were detected in T1 axial magnetic resonance imaging. The total muscle cross-sectional area of multifidus muscles (TMCSA) and the pure muscle cross-sectional area of multifidus muscles (PMCSA) were measured by a computerized analysis program, and the ratio of PMCSA to TMCSA (PMCSA/ TMCSA) was calculated.

Results There were no significant differences in TMCSA between the involved and the uninvolved sides in both groups. PMCSA was only significantly smaller at the S1 MSC on the involved side as compared with the uninvolved side in the L5 RAD group. The ratio of PMCSA to TMCSA was the lowest at the L5 MSP on the involved side in the L4 RAD group and at the S1 MSC on the involved side in the L5 RAD group.

Conclusion Our findings suggest that the most severe atrophy of multifidus muscle may occur at the mid-spinous process or mid-sacral crest level of the vertebra which is one level below the segmental number of the involved nerve root in patients with a single-level, unilateral lumbar radiculopathy.

Keywords Radiculopathy, Muscular atrophy, Electromyography, Magnetic resonance imaging

Received September 5, 2012; Accepted January 21, 2013

Corresponding author: In-Sik Lee

Department of Rehabilitation Medicine, Konkuk University Medical Center, 120 Neungdong-ro, Gwangjin-gu, Seoul 143-729, Korea

Tel: +82-2-2030-5347, Fax: +82-2-2030-5379, E-mail: mdlis@nate.com

@ This is an open-access article distributed under the terms of the Creative Commons Attribution Non-Commercial License (http://creativecommons. org/licenses/by-nc/3.0) which permits unrestricted noncommercial use, distribution, and reproduction in any medium, provided the original work is properly cited.

Copyright $\odot 2013$ by Korean Academy of Rehabilitation Medicine

\section{INTRODUCTION}

Paraspinal muscles play a role in maintaining the normal functions of the lumbar spine [1]. It is believed that low back pain [2-4], lumbosacral radiculopathy [5-8], and iatrogenic injury of medial branch of dorsal ramus after spinal surgery $[9,10]$ leads to atrophy and fatty infiltrations of paraspinal muscles. The paraspinal muscles can be analyzed based on histologic (size of muscle fibers) $[6,11,12]$, morphologic (cross-sectional area [CSA] of 
muscle or fat distribution in the muscle) $[2-5,7,8]$, and functional (physical strength) aspects [13,14]. In addition, atrophic changes and fatty infiltrations of the paraspinal muscles can be evaluated on computed tomography (CT) $[5,10]$, magnetic resonance imaging (MRI) $[2,4,7,8]$ and ultrasound imaging $[3,12]$.

Since multifidus muscle is unisegmentally innervated $[15,16]$, it serves as a clue for the electrodiagnosis of radiculopathy [17-19]. Campbell et al. [5] reported that focal atrophy and fatty replacement of right multifidus muscle was demonstrated on CT scans and MRI of the L5 level in a patient with the clinical and electrodiagnostic impression of the right L5 radiculopathy. Yoshihara et al. [6] reported that there were significant decreases in the size of type 1 and type 2 fibers in the L5 band of multifidus muscle on the affected side in patients with L5 nerve root compression by a disc herniation that had been identified during surgery. Hodges et al. [12] reported that CSA of multifidus muscle was reduced at the involved level after the experimental nerve root injury. Hyun et al. [7] reported asymmetric multifidus muscle atrophy occurred in patients with unilateral lumbosacral radiculopathy.

To date, however, no studies have described the difference in the location of multifidus atrophy according to the level of lumbar radiculopathy. Given the above background, we conducted this study to identify the correlations between the location of multifidus atrophy and the level of lumbar radiculopathy.

\section{MATERIALS AND METHODS}

\section{Subjects}

Of patients with a single-level, unilateral lumbar radiculopathy, those with L4 or L5 radiculopathy confirmed by electrodiagnosis between January 2007 and November 2011 were enrolled in the current retrospective study. This work was approved by the Institutional Review Board of Konkuk University Medical Center in 2012. The patients received a lumbosacral spine MRI before or after four weeks from the diagnosis of radiculopathy. Exclusion criteria were bilateral symptoms or signs of lower extremities, spinal fracture, spinal cord injury, spinal infection, spinal tumor, previous lumbosacral surgery, comorbidities that are influencing physical activities (e.g., cerebrovascular accident, severe heart disease, and severe musculoskeletal disease), and peripheral mononeuropathies or polyneuropathy affecting the results of

Table 1. Characteristics of subjects

\begin{tabular}{lcc}
\hline \multicolumn{1}{c}{ Characteristic } & $\begin{array}{c}\text { L4 RAD } \\
\text { group }(\mathbf{n = 1 0})\end{array}$ & $\begin{array}{c}\text { L5 RAD } \\
\text { group (n=27) }\end{array}$ \\
\hline Age (yr) & $49.80 \pm 14.94$ & $36.67 \pm 9.98$ \\
Gender (male:female) & $6: 4$ & $17: 10$ \\
Symptom duration (mo) & $9.2 \pm 12.36$ & $7.61 \pm 9.43$ \\
Involved side (right:left) & $5: 5$ & $12: 15$ \\
\hline
\end{tabular}

Values are presented as mean \pm standard deviation. RAD, radiculopathy.

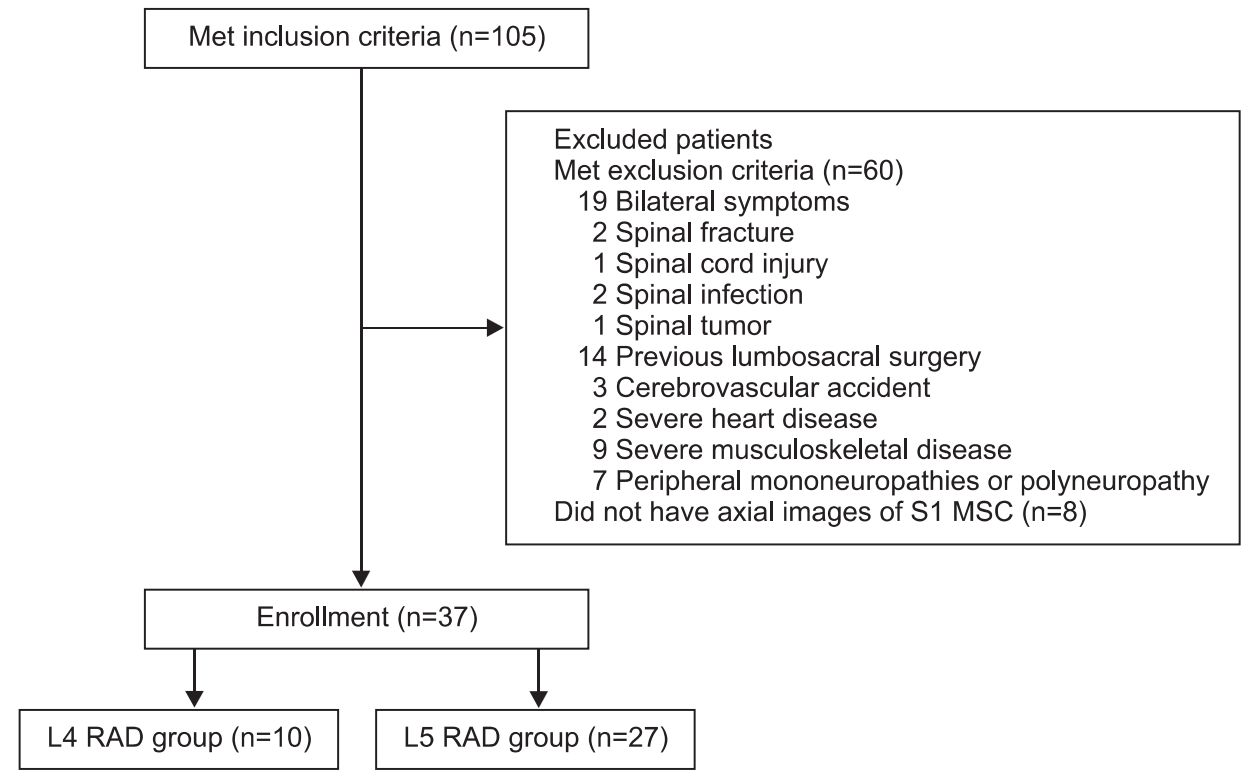

Fig. 1. A flowchart of subjects. S1 MSC, mid-sacral crest level of S1 vertebra; RAD, radiculopathy. 
electrophysiologic studies (Fig. 1). The electrodiagnostic criteria for lumbar radiculopathy was the detection of abnormal spontaneous activities at rest in the paraspinal muscles and/or involved lower limb muscles innervated by the same myotome but by different nerves, and normal findings in the lower limb muscles innervated by myotomes above and below the suspected lesion level. We therefore selected 37 patients who had a unilateral L4 or L5 radiculopathy for the current study. Then, we divided them into two groups: the L4 radiculopathy (L4 RAD, $\mathrm{n}=10$ ) group and the $\mathrm{L} 5$ radiculopathy (L5 RAD, $\mathrm{n}=27$ ) group (Table 1).

\section{Methods}

All subjects were retrospectively evaluated for electronic medical records on low back pain, affected side with radicular pain, duration of symptoms and results of electrodiagnosis. The T1-weighted fast spin-echo sequences of MRI were used for the evaluation of multifidus muscles. Axial planes were used to detect the CSA on the right and left multifidus muscles, for which the levels of analysis were the mid-spinous process level of L4 vertebra (L4 MSP), the mid-spinous process level of L5 vertebra (L5 MSP), and the mid-sacral crest level of S1 vertebra (S1 MSC) (Fig. 2).

We performed a quantitative analysis on the CSA of multifidus muscle by using the Rapidia analysis program (Infinitt, Seoul, Korea). The total muscle CSA of multifidus muscles (TMCSA) was measured by drawing manually the free regions of interest (ROI) over the boundary of the right and left multifidus muscles using a pen mouse, and the pure muscle CSA of multifidus muscles (PMCSA) was measured in such a manner that the fat component was eliminated within the ROI from the muscle by the threshold technique based on visual differences in pixel signal intensities (Fig. 2) [7,8]. The ratio of PMCSA to TMCSA (PMCSA/TMCSA) was calculated to estimate the severity of atrophy, thus suggesting that the smaller ratio was more severe with fatty infiltration and atrophy of multifidus muscle.

All CSAs were measured independently two times by two residents in the department of rehabilitation medicine, who were blinded to the clinical and electrophysiologic findings of patients.

\section{Statistical analysis}

Statistical analysis was performed using SPSS ver. 17.0 (SPSS Inc., Chicago, IL, USA). Shapiro-Wilk test was done
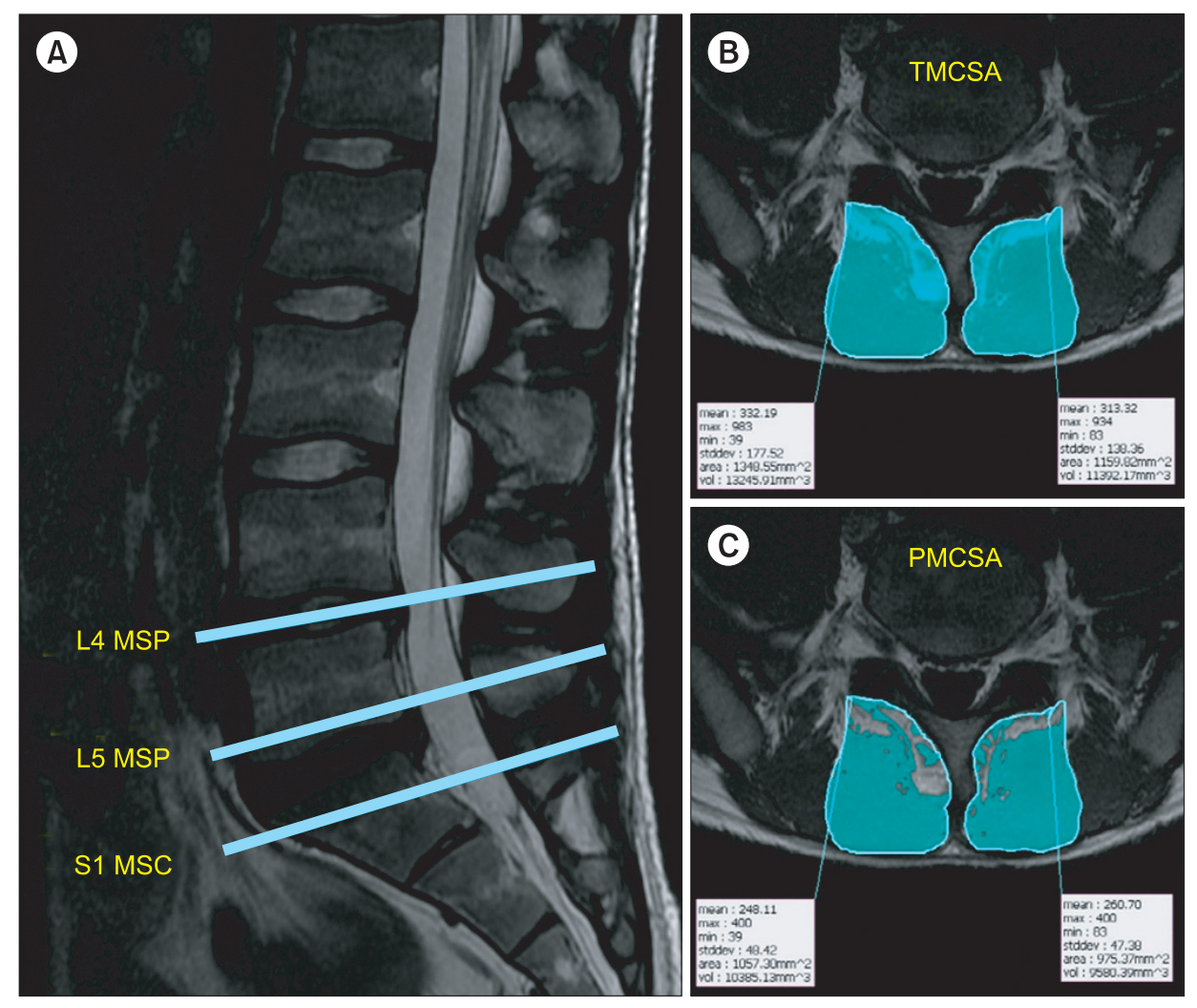

Fig. 2. Cross-sectional area (CSA) of multifidus muscles in a patient with right L5 radiculopathy. (A) shows axial planes at the mid-spinous process level of $\mathrm{L} 4$ vertebra (L4 MSP), the mid-spinous process level of L5 vertebra (L5 MSP), and the mid-sacral crest level of $\mathrm{S1}$ vertebra (S1 MSC). (B) indicates total muscle CSA of multifidus muscles (TMCSA) at the S1 MSC. (C) indicates pure muscle CSA of multifidus muscles (PMCSA) at the S1 MSC. 
to analyze the normal distribution of TMCSA, PMCSA, and PMCSA/TMCSA. Paired t-test was used to compare the parameters between the involved and uninvolved sides. Differences were considered significant when the p-value was less than 0.05 .

\section{RESULTS}

Overall, there were no significant differences in TMCSA between the involved and the uninvolved sides in both groups. In addition, there were no significant differences in PMCSA between the involved and the uninvolved sides in the L4 RAD group. PMCSA was significantly smaller only at the S1 MSC on the involved side as compared with the uninvolved side in the L5 RAD group $(\mathrm{p}=0.004)$ (Table 2).

PMCSA/TMCSA at the L4 MSP $(\mathrm{p}=0.028)$ and L5 MSP $(\mathrm{p}=0.001)$ was significantly lower on the involved side as compared with the uninvolved side in the L4 RAD group and at the L5 MSP $(\mathrm{p}=0.023)$ and S1 MSC $(\mathrm{p}<0.001)$ in the L5 RAD group. Finally, PMCSA/TMCSA was the lowest at the L5 MSP on the involved side in the L4 RAD group and at the S1 MSC on the involved side in the L5 RAD group (Table 2).

\section{DISCUSSION}

In the lumbar area, the multifidus muscle is formed by five separate bands, each of which arises from its particular lumbar spinous process of the L1 to L5 vertebrae [20]. Each band consists of several fascicles; from five at the L1 spinous process to one at the L5 spinous process [20]. They radiate caudally to insert into the mamillary processes of vertebrae two to five levels below, and the dorsal surface of iliac crest and sacrum (Fig. 3$)[16,20]$. The multifidus muscle is innervated by a single nerve root $[15,16]$. That is, each band of multifidus muscle receives its innervation from one dorsal ramus only [20]. Therefore, the multifidus inserting the same spinous process received innervation from the same medial branch of dorsal ramus, with a same segmental number of the spinous process, even if the origins of multifidus were different [21]. And then, each of the L1-L5 medial branches sent innervating nerves to multifidus via its dorsal surface at each midlamina, or mid-spinous process level of the vertebra with one level below the segmental number of dorsal ramus $[6,15,20-22]$. For instance, the fascicles of

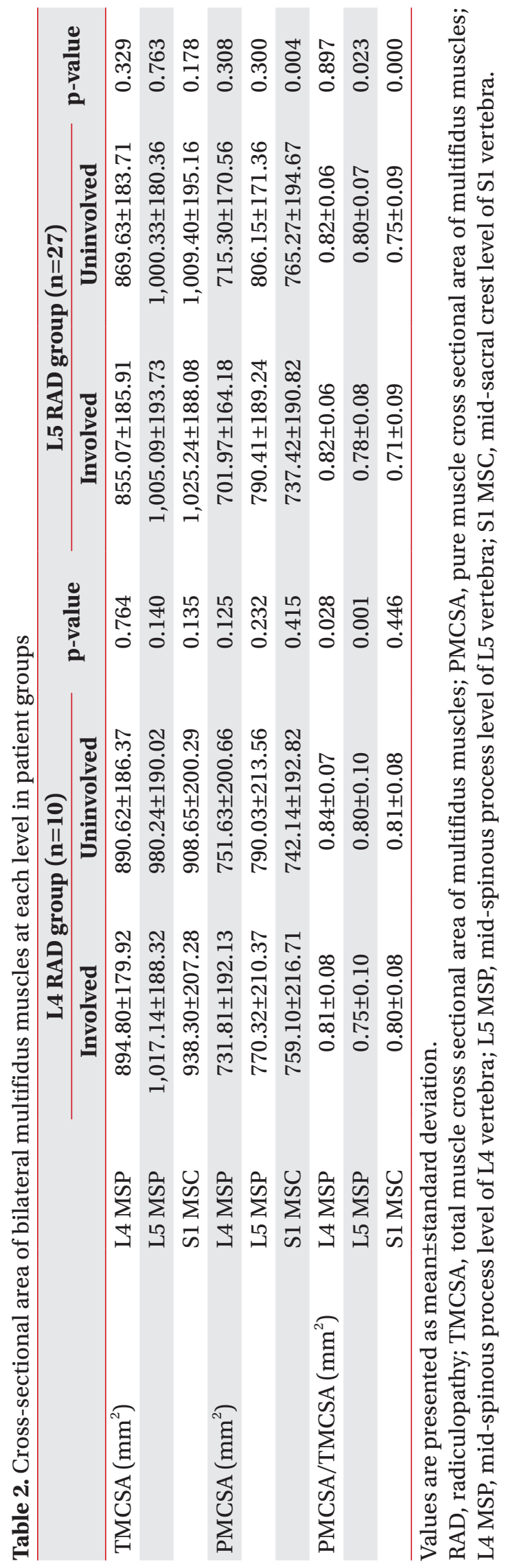

www.e-arm.org 


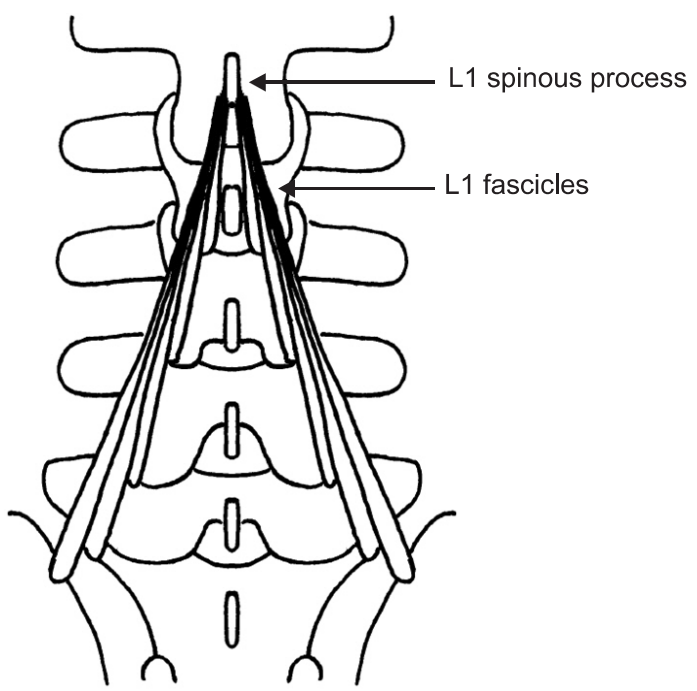

Fig. 3. A schematic illustration of the multifidus fascicles arising from the spinous process of Ll vertebra and innervated by Ll nerve root (L1 fascicles).

multifidus arise from the spinous process of L4 vertebra are innervated by L4 medial branch around the dorsal surface of L5 midlamina level, and the fascicles of multifidus arise from the spinous process of L5 vertebra are innervated by L 5 medial branch around the dorsal surface of S1 midlamina level. The fascicles originating from the higher spinous processes come to lie more superficially and laterally, therefore, in the most medial part of the groove between the lamina and spinous process, it is found that the fascicles arise from the spinous process one level above (Fig. 4) [20]. For example, the fascicles innervated by L4 nerve root (L4 fascicles) lie most medially and deeply, while fascicles innervated higher nerve roots (L1-L3 fascicles) lie laterally and superficially at the dorsal surface of lamina and spinous process of the L5 vertebra (Fig. 4). Therefore, L4 fascicles appose the spinous process of the L5 vertebra, and L5 fascicles appose the median sacral crest of the S1 vertebra.

Previous studies have reported the multifidus atrophy in patients with radiculopathy; Bae et al. [4] reported that the disc herniation and nerve root compression had no correlations with multifidus atrophy. In addition, Hyun et al. [7] reported that there was a significant asymmetric atrophy of the ipsilateral multifidus muscles only at L4-L5 and L5-S1 intervertebral disc levels in patients with unilateral lumbosacral radiculopathy. Moreover, Chae et al. [8] reported that cervical multifidus muscle atrophy was predominantly found below the level of radiculopathy in

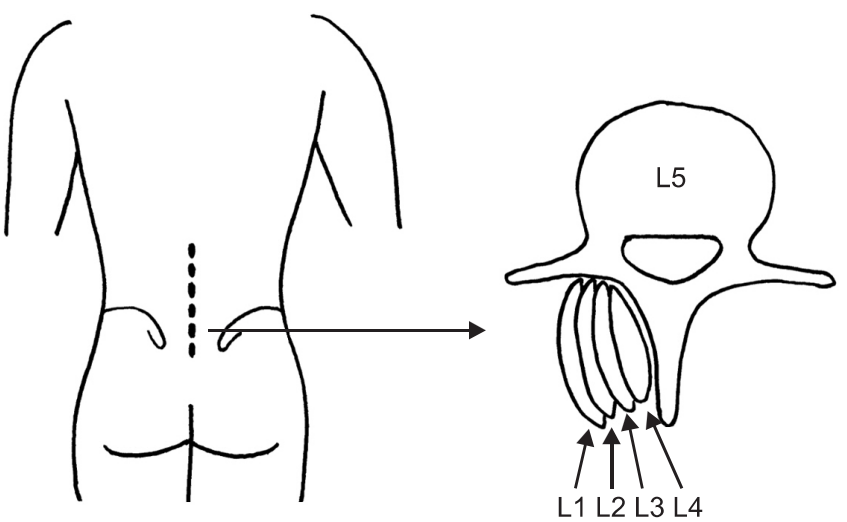

Fig. 4. A schematic illustration of the multifidus fascicles at the mid-spinous process level of L5 vertebra (L5 MSP). The fascicles innervated by L4 nerve root (L4 fascicles) appose the spinous process of L5 vertebra.

patients with unilateral cervical radiculopathy. However, these authors did not define the levels of radiculopathy but included polyradiculopathy. In addition, they selected the axial planes in the intervertebral disc space to analyze the CSA of multifidus muscles with no respect to the anatomy of innervation. Therefore, they failed to identify the correlations between the location of multifidus atrophy and the level of radiculopathy. In the current study, we used axial planes of mid-spinous process level or mid-sacral crest level in measuring the CSA of multifidus muscles considering the anatomical innervation site in it.

Our results showed that there were no significant differences in the TMCSA between the involved and the uninvolved sides in both groups. However, most of TMCSAs were greater on the involved side as compared with the uninvolved side. Such results indicate that the pseudohypertrophy of muscle may also occur [23], although the atrophy and fatty replacement is the typical finding of denervation. It is therefore difficult to determine whether the multifidus atrophy occurred solely based on TMCSA with having no relations to the fat infiltration.

In both groups, PMCSA was smaller on the involved side as compared with the uninvolved side, but this reached a statistical significance only at the S1 MSC in the L5 RAD group. It may be due to a discrepancy in the PMCSA between the both sides. If patients have a greater PMCSA on the involved side as compared with the uninvolved side prior to the onset of the lumbar radiculopathy, they would show no significant differences in the PMCSA between both sides or have a greater PMCSA on 
the involved side as compared with the uninvolved side although they have a multifidus atrophy on the involved side after the onset of the lumbar radiculopathy. We therefore calculated PMCSA/TMCSA to estimate the severity of atrophy, because it is difficult to decide whether the multifidus atrophy occurred solely based on PMCSA.

In this study, PMCSA/TMCSA was the lowest at the L5 MSP on the involved side in the L4 RAD group and at the S1 MSC on the involved side in the L5 RAD group. These results showed that the most severe atrophy of multifidus muscle was seen at the mid-spinous process level or midsacral crest level of the vertebra which is one level below the segmental number of the involved nerve root in patients with L4 or L5 radiculopathy.

The limitation of this study is that the number of patients was small especially in the L4 RAD group. In the current study, we excluded eight patients whose axial images of S1 MSC were not available (Fig. 1). This implies that the axial images of S1 MSC are needed to evaluate the multifidus muscle that is innervated by L 5 nerve root. We did not evaluate patients with S1 radiculopathy, because Bogduk [24] and Macintosh et al. [16] have reported that the multifidus muscle is not innervated by the S1 nerve root. Since we enrolled the patients with unilateral L4 or L5 radiculopathy, we could not investigate the patterns of multifidus atrophy in patients with bilateral symptoms, other levels of single radiculopathy, or polyradiculopathy. Further studies are therefore warranted to delineate the mechanisms by which the multifidus atrophy occurs in these patients.

In conclusion, our findings suggest that the most severe atrophy of multifidus muscle may occur at the midspinous process level or mid-sacral crest level of the vertebra which is one level below the segmental number of the involved nerve root in patients with a single-level, unilateral lumbar radiculopathy. Based on our study results, we propose that clinicians evaluate the severity of multifidus atrophy and identify its location, as well as the disc herniation and nerve root compression on MRI, which may be of help for not only making a diagnosis the denervation atrophy of lumbar radiculopathy but also for determining its level.

\section{CONFLICT OF INTEREST}

No potential conflict of interest relevant to this article was reported.

\section{ACKNOWLEDGMENTS}

This paper was written as part of Konkuk University's research support program for its faculty on sabbatical leave in 2012.

\section{REFERENCES}

1. Kong WZ, Goel VK, Gilbertson LG, Weinstein JN. Effects of muscle dysfunction on lumbar spine mechanics: a finite element study based on a two motion segments model. Spine (Phila Pa 1976) 1996;21:2197-206.

2. Parkkola R, Rytokoski U, Kormano M. Magnetic resonance imaging of the discs and trunk muscles in patients with chronic low back pain and healthy control subjects. Spine (Phila Pa 1976) 1993;18:830-6.

3. Hides JA, Stokes MJ, Saide M, Jull GA, Cooper DH. Evidence of lumbar multifidus muscle wasting ipsilateral to symptoms in patients with acute/subacute low back pain. Spine (Phila Pa 1976) 1994;19:165-72.

4. Bae JH, Na JK, Yu JY, Park YO. Atrophy of multifidus muscle on low back pain patients. J Korean Acad Rehabil Med 2001;25:684-91.

5. Campbell WW, Vasconcelos O, Laine FJ. Focal atrophy of the multifidus muscle in lumbosacral radiculopathy. Muscle Nerve 1998;21:1350-3.

6. Yoshihara K, Shirai Y, Nakayama Y, Uesaka S. Histochemical changes in the multifidus muscle in patients with lumbar intervertebral disc herniation. Spine (Phila Pa 1976) 2001;26:622-6.

7. Hyun JK, Lee JY, Lee SJ, Jeon JY. Asymmetric atrophy of multifidus muscle in patients with unilateral lumbosacral radiculopathy. Spine (Phila Pa 1976) 2007;32:E598-602.

8. Chae SH, Lee SJ, Kim MS, Kim TU, Hyun JK. Cervical multifidus muscle atrophy in patients with unilateral cervical radiculopathy. J Korean Acad Rehabil Med 2010;34:743-51.

9. Sihvonen T, Herno A, Paljarvi L, Airaksinen O, Partanen J, Tapaninaho A. Local denervation atrophy of paraspinal muscles in postoperative failed back syndrome. Spine (Phila Pa 1976) 1993;18:575-81.

10. Hyun SJ, Kim YB, Kim YS, Park SW, Nam TK, Hong HJ, et al. Postoperative changes in paraspinal muscle volume: comparison between paramedian interfascial and midline approaches for lumbar fusion. J Korean Med Sci 2007;22:646-51. 
11. Zhao WP, Kawaguchi Y, Matsui H, Kanamori M, Kimura T. Histochemistry and morphology of the multifidus muscle in lumbar disc herniation: comparative study between diseased and normal sides. Spine (Phila Pa 1976) 2000;25:2191-9.

12. Hodges P, Holm AK, Hansson T, Holm S. Rapid atrophy of the lumbar multifidus follows experimental disc or nerve root injury. Spine (Phila Pa 1976) 2006; 31:2926-33.

13. Estlander AM, Vanharanta H, Moneta GB, Kaivanto K. Anthropometric variables, self-efficacy beliefs, and pain and disability ratings on the isokinetic performance of low back pain patients. Spine (Phila Pa 1976) 1994;19:941-7.

14. Mannion AF, Dolan P. Electromyographic median frequency changes during isometric contraction of the back extensors to fatigue. Spine (Phila Pa 1976) 1994; 19:1223-9.

15. Bogduk N, Wilson AS, Tynan W. The human lumbar dorsal rami. J Anat 1982;134(Pt 2):383-97.

16. Macintosh JE, Valencia F, Bogduk N, Munro RR. The morphology of the human lumbar multifidus. Clin Biomech 1986;1:196-204.

17. Haig AJ, Moffroid M, Henry S, Haugh L, Pope M. A technique for needle localization in paraspinal muscles with cadaveric confirmation. Muscle Nerve 1991;
14:521-6.

18. Haig AJ, Talley C, Grobler LJ, LeBreck DB. Paraspinal mapping: quantified needle electromyography in lumbar radiculopathy. Muscle Nerve 1993;16:477-84.

19. Kim BJ, Date ES, Derby R, Lee SH, Seo KS, Oh KJ, et al. Electromyographic technique for lumbar multifidus examination: comparison of previous techniques used to localize the multifidus. Arch Phys Med Rehabil 2005;86:1325-9.

20. Kalimo H, Rantanen J, Viljanen T, Einola S. Lumbar muscles: structure and function. Ann Med 1989;21: 353-9.

21.Zhang J, Tsuzuki N, Hirabayashi S, Saiki K, Fujita K. Surgical anatomy of the nerves and muscles in the posterior cervical spine: a guide for avoiding inadvertent nerve injuries during the posterior approach. Spine (Phila Pa 1976) 2003;28:1379-84.

22. Lau P, Mercer S, Govind J, Bogduk N. The surgical anatomy of lumbar medial branch neurotomy (facet denervation). Pain Med 2004;5:289-98.

23. Petersilge CA, Pathria MN, Gentili A, Recht MP, Resnick D. Denervation hypertrophy of muscle: MR features. J Comput Assist Tomogr 1995;19:596-600.

24. Bogduk N. A reappraisal of the anatomy of the human lumbar erector spinae. J Anat 1980;131(Pt 3):525-40. 